\title{
Associação entre aleitamento materno e excesso de peso em pré-escolares
}

\author{
Association between breastfeeding and overweight in preschoolers \\ Relación entre la lactancia materna y el exceso de peso en niños preescolares \\ Rivaldo da Costa Macêdo ${ }^{1}$ io https://orcid.org/0000-0003-1107-5668 \\ Carmen Viana Ramos ${ }^{1}$ io https://orcid.org/0000-0003-3378-0149 \\ Adriana de Azevedo Paiva² ${ }^{2}$ is htpp://orcid.org/0000-0003-4843-4572 \\ Maria do Carmo de Carvalho e Martins ${ }^{1}$ io https://orcid.org/0000-0002-6009-3793 \\ Camila Aparecida Pinheiro Landim Almeida ${ }^{1}$ io https:/orcid.org/0000-0002-1667-9957 \\ Suzana Maria Rebêlo Sampaio da Paz² io nttps://orcid.orgy/0000-0001-6681-8081
}

Como citar:

Macêdo RC, Ramos CV, Paiva AA, Martins MC, Almeida CA, Paz SM. Associação entre aleitamento materno e excesso de peso em pré-escolares. Acta Paul Enferm. 2020;33:AAPE20190025.

DOI http://dx.doi.org/10.37689/actaape/2020A00025

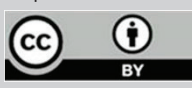

Descritores
Obesidade pediátrica; Pré-escolar; Aleitamento
materno

Keywords

Pediatric obesity; Child, preschool; Breast feeding

Descriptores

Obesidad pediátrica; Preescolar; Lactancia materna

Submetido 5 de Fevereiro de 2019

Aceito

16 de Outubro de 2019

Autor correspondente

Rivaldo da Costa Macêdo

E-mail: ricomamede@bol.com.br

\section{Resumo}

Objetivo: Analisar associação entre aleitamento materno e excesso de peso em pré-escolares.

Métodos: Estudo transversal com 448 pré-escolares de 10 Centros Municipais de Educação Infantil do município de Teresina-PI. Realizou-se reunião com os pais, momento em que foi aplicado o questionário estruturado com questões relativas aos dados sociodemográficos e alimentação pregressa das crianças. Posteriormente, foram obtidas as medidas antropométricas das crianças (peso e altura). Foi considerado excesso de peso (sobrepeso + obesidade) quando o resultado do escore- $z$ foi maior que ou igual a +2 , e os menores que +2 foram classificados como sem excesso de peso. A análise da associação entre 0 aleitamento materno exclusivo até seis meses, aleitamento exclusivo até quatro meses e 0 aleitamento materno com 0 estado nutricional da criança foi feita utilizando-se o teste qui-quadrado de Pearson $\left(\chi^{2}\right)$, enquanto a razão de prevalência (RP) foi estimada para quantificar as associações entre 0 aleitamento materno e 0 estado nutricional controlado pelas variáveis sociodemográficas. Todas as estimativas e os intervalos de confiança de 95\% foram calculados utilizando o modelo de regressão de Poisson com variância robusta.

Resultados: A prevalência do excesso de peso e a proporção das crianças que receberam 0 aleitamento materno exclusivo até os 6 meses de idade foi de 11,16\% e 41,9\%, respectivamente. 0 excesso de peso foi 2,5 vezes mais elevado entre as crianças não amamentadas (IC 95\%: 1,09; 5,83); e, após o controle das variáveis sociodemográficas, verificou-se que as crianças maiores de 48 meses tiveram risco elevado para 0 excesso de peso (RP: 1,69; IC 95\%: 1,01; 2,85) em relação aos menores de 48 meses de idade ( $p=0,04)$.

Conclusão: 0 presente estudo demonstrou que as crianças que receberam aleitamento materno foram protegidas contra o excesso de peso.

\section{Abstract}

Objective: To analyze association between breastfeeding and overweight in preschoolers.

Methods: A cross-sectional study with 448 preschoolers from 10 Municipal Centers of Early Childhood Education in the city of Teresina, state of Piauí. A meeting was held with the parents, at which time the structured questionnaire with questions related to sociodemographic data and past feeding of the children was applied. Subsequently, the anthropometric measurements of the children (weight and height). Overweight (overweight + obesity) was considered when the $z$-score result was greater than or equal to +2 . Children younger than +2 were classified as not overweight. The association between exclusive breastfeeding up to six months, exclusive breastfeeding up to four months and breastfeeding with the child's nutritional status was analyzed using Pearson's chi-square test $\left(\chi^{2}\right)$, while the prevalence ratio (PR) was estimated to quantify the associations between breastfeeding and nutritional status controlled by sociodemographic variables. All 
estimates and $95 \%$ confidence intervals were calculated using the robust variance Poisson regression model.

Results: The prevalence of overweight and the proportion of children who received exclusive breastfeeding until 6 months of age were $11.16 \%$ and $41.9 \%$, respectively. Overweight was 2.5 times higher among non-breastfed children ( $95 \% \mathrm{Cl}: 1.09 ; 5.83)$. After controlling for sociodemographic variables, it was found that children older than 48 months were at high risk for overweight (PR: $1.69 ; 95 \% \mathrm{Cl}: 1.01 ; 2.85)$ compared to children younger than 48 months. months old ( $p=0.04)$.

Conclusion: The present study demonstrated that breastfed newborns were protected against overweight.

\section{Resumen}

Objetivo: Analizar la elación entre la lactancia materna y el exceso de peso en niños preescolares.

Métodos: Estudio transversal con 448 niños preescolares de 10 Centros Municipales de Educación Infantil del municipio de Teresina, estado de Piaúi. Se realizó una reunión con los padres, momento en que se aplicó el cuestionario estructurado con preguntas relativas a datos sociodemográficos y alimentación anterior de los niños. Posteriormente, se obtuvieron las medidas antropométricas de los niños (peso y estatura). Se consideró exceso de peso (sobrepeso + obesidad) cuando el resultado de la puntuación z fue mayor 0 igual a +2; y los menores a +2 se clasificaron como sin exceso de peso. El análisis de la relación entre lactancia materna exclusiva hasta los seis meses, lactancia exclusiva hasta los cuatro meses y lactancia materna con el estado nutricional del niño se realizó mediante la prueba $c^{2}$ de Pearson. Para cuantificar las relaciones entre la lactancia materna y el estado nutricional controlado por las variables sociodemográficas se calculó la a razón de prevalencia (RP). Todas las estimaciones y los intervalos de confianza de 95\% se calcularon utilizando el modelo de regresión de Poisson con varianza robusta.

Resultados: La prevalencia del exceso de peso y la proporción de los niños que recibieron lactancia materna exclusiva hasta los 6 meses de edad fue de $11,16 \%$ y $41,9 \%$, respectivamente. El exceso de peso fue 2,5 veces más elevado en niños no amamantados (IC 95\%: 1,09; 5,83). Luego del control de las variables sociodemográficas, se verificó que los niños mayores de 48 meses tuvieron un riesgo elevado de exceso de peso (RP: 1,69; IC 95\%: 1,01; 2,85) con relación a los menores de 48 meses de edad $(p=0,04)$.

Conclusión: El presente estudio demostró que los niños que recibieron lactancia materna fueron protegidos contra el exceso de peso.

\section{Introdução}

A obesidade é caracterizada pelo acúmulo de gordura corporal em excesso, com prejuízo à saúde em todas as idades — sobretudo nos primeiros anos de vida —, e tem incidência e prevalência crescentes. ${ }^{(1,2)}$ Dados da Organização Mundial da Saúde (OMS) apontam que, no mundo, cerca de 42 milhóes de crianças menores de 5 anos apresentaram sobrepeso em 2013. ${ }^{(3)}$ No Brasil e no Estado do Piaui ${ }^{(4)}$ os estudos recentes apontam prevalências de 15,8\% e 14,2\%, respectivamente.

A pré-escola corresponde ao período de 2 a 6 anos de idade, sendo esse um período crítico na vida da criança, em que se torna necessário e importante a sedimentação de hábitos alimentares saudáveis, uma vez que essa é uma fase de transiçấo: a criança sai de uma fase de total dependência (lactentes) para entrar em uma fase de maior independência (escolar e adolescência). A construçáo de um hábito alimentar saudável é capaz de proporcionar crescimento e desenvolvimento adequados, evitar déficits nutricionais e ainda prevenir os agravos à saúde como hipertensão arterial sistêmica, diabetes tipo 2, doença cardiovascular, osteoporose e obesidade. ${ }^{(5)}$ Assim, esses hábitos devem ser constituídos ainda no primeiros anos, como é o caso do aleitamento materno continuado até os 2 anos ou mais de idade. ${ }^{(6)}$
A $\mathrm{OMS}^{(7)}$ recomenda manter o AME até os 6 meses de vida e complementado até os 2 anos ou mais de idade, contudo a II Pesquisa de Prevalência de Aleitamento Materno realizada nas Capitais Brasileiras e no Distrito Federal (PPAM -2008) verificou que a prevalência do AME foi de $41 \%$ e $43,7 \%$ no Brasil e em Teresina, respectivamente. ${ }^{(8)}$ Entre as principais causas da baixa adesão ao $\mathrm{AME}$ são a menor escolaridade materna, o retorno precoce ao trabalho, a falta de apoio durante o ciclo gravídico-puerperal e as questóes subjetivas como sentimentos de derrota e frustração. ${ }^{(9-1)}$

Desse modo, é necessário corrigir tais distorçôes e assegurar condições para que as crianças recebam o aleitamento materno conforme preconizado pela OMS, encontrando um ponto de entrada para acesso às mães e crianças. ${ }^{(12)}$ Nesse sentido, o ambiente da pré -escola mostra-se atrativo e ideal para investigar a ocorrência de distúrbios nutricionais como o excesso de peso nas crianças, bem como para propor estratégias de intervenção visando a melhoria dessas condiçôes.

Assim, tendo em vista o fato de a idade pré-escolar ser um momento crucial para prevençáo de distúrbios alimentares, associado à possibilidade de intervenção no ambiente escolar e à inexistência de estudos abrangentes realizados em Teresina para levantamento dessa problemática, objetivamos 
investigar a associação entre aleitamento materno e excesso de peso nos pré-escolares e testar a hipótese de que o aleitamento materno, como primeiro alimento saudável, é capaz de oferecer proteção contra o excesso de peso nos pré-escolares.

\section{Métodos}

Trata-se de um estudo transversal, tendo como público crianças na idade de 3 a 6 anos incompletos, matriculadas nos Centros Municipais de Educação Infantil (CMEI) da zona urbana do município de Teresina, capital do Piauí. De acordo com a Secretaria Municipal de Educação (SEMEC), em 2015, havia 83.003 alunos matriculados na rede municipal de ensino, sendo que 12.806 dessas crianças eram pré-escolares e estavam distribuídas em 143 CMEIs. ${ }^{(13)}$

O cálculo amostral foi feito utilizando fórmula de Levin, ${ }^{(14)}$ com perda amostral de $20 \%$, erro amostral de $5 \%$ para o grau de confiança de $95 \%$. $\mathrm{O}$ poder do teste utilizado para cálculo da amostra foi $80,43 \%$. A amostra final foi constituída de 448 crianças. Os dados foram coletados no período de setembro a outubro de 2016 . Não houve perdas nem recusas à participação no estudo.

A seleção das crianças foi feita por meio de amostra probabilística proporcional ao número de alunos matriculados na pré-escola de cada uma das quatro zonas da cidade (Zona Norte, Zona Leste, Zona Sul e Zona Sudeste), e o sorteio foi de forma simples. Assim, cada zona da cidade teve pelo menos duas escolas sorteadas de forma a alcançar a quantidade de alunos matriculados para a aplicação dos questionários. Entretanto, quando a quantidade de alunos proporcional à zona não foi suficiente, uma nova escola foi sorteada até que o número amostral mínimo de participantes fosse atingido.

Desse modo, a pesquisa foi realizada em 10 escolas situadas na zona urbana do município. Foram considerados critérios de inclusão na pesquisa: ter idade entre 36 e 59 meses e estar matriculado e frequentando regularmente as escolas; não ter diagnóstico de doenças que pudesse interferir nos resultados do estudo, como síndrome de Prader Willie, Down, hipotireoidismo e o uso crônico de corticoides.
Como estratégia para coletar os dados da pesquisa, realizou-se uma reunião prévia com os pais ou responsáveis em cada escola a fim de esclarecer os objetivos do estudo. Dessa forma, aqueles que concordaram em participar da pesquisa assinaram o Termo de Consentimento Livre e Esclarecido (TCLE) e responderam ao questionário da pesquisa. $\mathrm{Na}$ oportunidade, foram dadas orientaçóes sobre os cuidados de saúde e nutrição das crianças, bem como realizada uma avaliação pediátrica dos alunos pelo pesquisador responsável. A avaliação antropométrica das crianças foi realizada no final da reunião.

$\mathrm{O}$ instrumento utilizado para a coleta de dados consistiu em um questionário estruturado com questôes relativas aos dados sociodemográficos das mães ou responsáveis e alimentação pregressa das crianças, questóes, estas, relacionadas à prática de aleitamento materno. $\mathrm{O}$ referido instrumento foi adaptado do questionário validado na pesquisa realizada por Caldeira. ${ }^{(15)}$

As variáveis antropométricas utilizadas no estudo foram o peso e a altura, coletadas por uma equipe previamente treinada, composta por uma supervisora nutricionista e dois acadêmicos do curso de nutrição. As crianças foram pesadas com roupas leves e descalças, conforme as instruçóes do manual do SISVAN (Ministério da Saúde) para a coleta de dados antropométricos. ${ }^{(11)}$ Utilizou-se balança digital com capacidade de $180 \mathrm{~kg}$, da marca Avanutri; e estadiômetro portátil, com graduação $0,1 \mathrm{~cm}$ e escala de $20 \mathrm{~cm}$ a $200 \mathrm{~cm}$, também da marca Avanutri, equipamentos para avaliação, fabricados na cidade de Três Rios, Rio de Janeiro,Brasil.

Neste estudo, utilizou-se como parâmetro para avaliação do estado nutricional o IMC/Idade, conforme definição da OMS, ${ }^{(16)}$ recomendados pelo Ministério da Saúde, ${ }^{(17)}$ por ser um índice muito utilizado para identificar o excesso de peso entre crianças. ${ }^{(15,18)}$ A classificação foi feita segundo o escore-z: resultado maior ou igual +2 foi considerado "com excesso de peso (sobrepeso + obesidade)", e os resultados menores que +2 foram classificados na categoria "sem excesso de peso". Dessa forma, foi considerada a variável de desfecho o diagnóstico nutricional da criança, com duas categorias: sem excesso de peso e com excesso de peso. A classificação do estado nutricional foi feita pelo nutricionista supervisor de 
campo conforme as tabelas contidas no manual do Sisvan $^{(17)}$ do Ministério da Saúde (2011) e revisada pelos pesquisadores responsáveis pelo estudo.

As variáveis sociodemográficas foram utilizadas para descrever a amostra e controlar o efeito do aleitamento sobre o excesso de peso das crianças. Foram consideradas as variáveis da mãe: idade da mãe ( $\leq 36$ e > 36 anos), trabalho materno (sim e não), renda familiar mensal ( $\leq$ 1 salário mínimo e $>1$ salário mínimo). Quanto às variáveis da criança, utilizou-se: sexo (masculino e feminino), peso ao nascer $(<2.500 \mathrm{~g} \mathrm{e} \geq 2.500 \mathrm{~g})$, idade da criança ( $\leq 4$ anos e $>4$ anos). No que tange ao tipo de aleitamento, foram utilizadas as variáveis categorizadas relacionadas ao aleitamento materno exclusivo (AME) a menores de 4 meses (sim e não), aleitamento materno exclusivo a menores de 6 meses (sim e não) e aleitamento materno (AM) (sim e não).

As definiçóes de aleitamento materno adotadas neste estudo seguiram as recomendaçóes contidas no documento intitulado Indicators for assessing infant and young child feeding practices, da OMS, ${ }^{(19)}$ que considera como aleitamento materno exclusivo quando a criança recebe leite materno (incluindo leite materno ordenhado), permitindo ela receba soro oral, vitaminas e medicamentos, sem a inclusão de qualquer outro líquido ou alimento; e considera-se aleitamento materno quando a criança recebe leite materno (incluindo leite materno ordenhado), podendo receber também qualquer outro líquido ou alimento, incluindo outros leites e fórmulas infantis.

Os dados foram tabulados na planilha do software Microsoft Office Excel e analisados utilizando o pacote estatístico Stata ${ }^{\circledR}$, versão 12. A análise da associação entre o aleitamento materno exclusivo até 6 meses, aleitamento exclusivo até 4 meses e o aleitamento materno com o estado nutricional (sem excesso de peso e com excesso de peso) atual da criança foi feita utilizando-se o teste qui-quadrado de Pearson $\left(\chi^{2}\right)$ ou teste exato de Fisher (utilizado quando a frequência for inferior a 5), quando apropriado. A razão de prevalência $(\mathrm{RP})$ foi calculada para quantificar as associaçôes entre as práticas de aleitamento materno e o estado nutricional. Todas as estimativas e intervalos de confiança de 95\% (IC 95\%) foram calculados utilizando modelo de regressão de Poisson com variância robusta. ${ }^{(20)}$ As RPs e seus ICs 95\% foram ajus- tados num modelo que considerou o estado nutricional como variável dependente e o AME e AM como variáveis independentes, sendo controladas pelas variáveis sociodemográficas. As variáveis com valor de $p<0,10$ foram colocadas todas em um modelo final. Foram aceitos como estatisticamente significativos os testes com valor de $p<0,05$.

$\mathrm{O}$ projeto de pesquisa foi autorizado pelo Comitê de Ética em Pesquisa (CEP) do Centro Universitário Uninovafapi por meio do parecer $\mathrm{n}^{\mathrm{o}}$ 1.673.624/2016, de 11 de agosto de 2016, conforme recomenda a Resolução 466/12 do Conselho Nacional de Saúde (CNS) do Ministério da Saúde (MS). Os pais ou responsáveis assinaram o TCLE.

\section{Resultados}

Participaram do estudo 448 crianças, sendo 230 $(51,3 \%)$ do sexo masculino. No que se refere ao peso ao nascer, $37(8,3 \%)$ foram de baixo peso $(<2.500 \mathrm{~g})$, e a maioria $(61,2 \%)$ tinham menos de 4 anos. Quanto as características maternas, $391(87,3 \%)$ possuíam idade inferior a 36 anos, $302(67,4 \%)$ não trabalhavam e $395(79,2 \%)$ tinham renda familiar inferior a um salário mínimo (Tabela 1). O percentual de crianças amamentadas foi de 95,7\% (429). Destas, 194 (43,3\%) receberam aleitamento materno exclusivo até os 4 meses e $188(41,9 \%)$ até os 6 meses de vida.

Tabela 1. Distribuição da amostra, segundo variáveis sociodemográficas da criança e maternas de pré-escolares

\begin{tabular}{lc}
\hline Características & $\mathbf{n}(\%)$ \\
\hline Sexo & \\
$\quad$ Masculino & $230(51,3)$ \\
$\quad$ Feminino & $218(48,7)$ \\
Peso ao nascer (gramas) & \\
$\quad<2.500$ & $37(8,3)$ \\
$\geq 2.500$ & $411(91,7)$ \\
Idade (anos) & \\
$\leq 4$ & $274(61,2)$ \\
$>4$ & $174(38,8)$ \\
Idade da mãe (anos) & \\
$\leq 36$ & $391(87,3)$ \\
$>36$ & $57(12,7)$ \\
Trabalho materno atual & \\
$\quad$ Sim & $146(32,6)$ \\
Não & $302(67,4)$ \\
Renda familiar (salários mínimos) & \\
$\leq 1$ & $395(79,2)$ \\
$>1$ & $93(20,8)$ \\
\hline
\end{tabular}


A figura 1 apresenta os resultados da avaliação nutricional dos pré-escolares, mostrando que a prevalência de excesso de peso entre eles foi de 11,2\% (escore- $z$ $>+2$ ), sendo $6,9 \%$ de sobrepeso e $3,8 \%$ de obesidade.

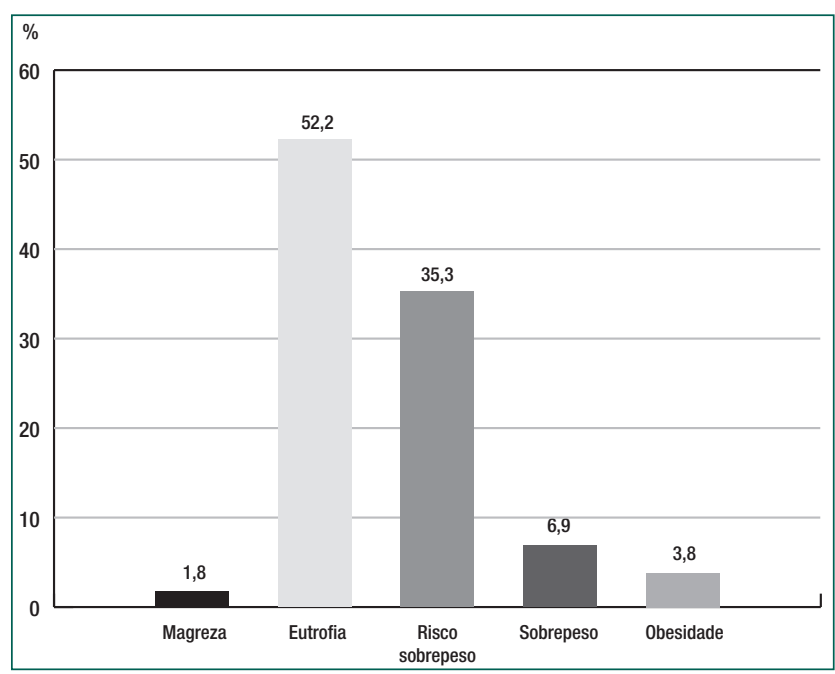

Figura 1. Estado nutricional, segundo o IMC/Idade, em préescolares

A tabela 2 mostra a relação entre a prática do aleitamento materno e a prevalência de excesso de peso, demonstrando que apenas $10,5 \%$ das crianças que mamaram apresentaram excesso de peso; enquanto $26,3 \%$ dos pré-escolares que não receberam leite materno desenvolveram excesso de peso. Dessa forma, a criança que não foi amamentada teve 2,5 vezes mais chances de ter excesso de peso quando comparada com quem recebeu o aleitamento (IC 95\%: 1,09; 5,83). O aleitamento materno exclusivo até os 6 meses e o aleitamento materno até os 4 meses não mostrou associação estatisticamente significativa com o excesso de peso (Tabela 2).

Tabela 2. Associação entre variáveis de aleitamento materno e excesso de peso em pré-escolares

\begin{tabular}{|c|c|c|c|c|}
\hline Variáveis & $\begin{array}{c}\text { Sem } \\
\text { excesso de } \\
\text { peso } \\
n(\%)\end{array}$ & $\begin{array}{c}\text { Com } \\
\text { excesso de } \\
\text { peso } \\
\text { n(\%) }\end{array}$ & $\mathrm{P}\left(\chi^{2}\right)$ & $\begin{array}{l}\text { Razão de } \\
\text { prevalência } \\
\text { (IC 95\%) }\end{array}$ \\
\hline \multicolumn{5}{|c|}{ Aleitamento materno } \\
\hline Sim & $384(89,5)$ & $45(10,5)$ & 0,032 & 1,00 \\
\hline Não & $14(73,7)$ & $5(26,3)$ & & $2,50(1,12 ; 5,60)$ \\
\hline \multicolumn{5}{|c|}{$\begin{array}{l}\text { Aleitamento materno exclusivo } \\
(<6 \text { meses })\end{array}$} \\
\hline Sim & $167(88,8)$ & $21(11,2)$ & 0,996 & 1,00 \\
\hline Não & $231(88,8)$ & $29(11,2)$ & & $0,99(0,59 ; 1,70)$ \\
\hline \multicolumn{5}{|c|}{$\begin{array}{l}\text { Aleitamento materno exclusivo } \\
(<4 \text { meses })\end{array}$} \\
\hline $\operatorname{Sim}$ & $172(88,7)$ & $22(11,3)$ & 0,916 & 1,00 \\
\hline Não & $226(89,0)$ & $28(11,0)$ & & $0,97(0,59 ; 1,70)$ \\
\hline
\end{tabular}

A Regressão de Poisson, mesmo após a introdução das varáveis no modelo, demonstrou associação entre aleitamento materno e excesso de peso com elevada chance de desenvolver excesso de peso entre as crianças não amamentadas (RP: 2,5; IC 95\%: 1,08; $5,56)$ e, além disso, verificou-se maior chance do de excesso de peso nas crianças maiores de 4 anos de idade (RP: 1,7; IC 95\%: 1,01; 2,85) quando comparado com os menores de 4 anos $(p=0,04)$ (Tabela 3$)$.

Tabela 3. Modelo final dos fatores associados ao excesso de peso em pré-escolares

\begin{tabular}{lccccc}
\hline Variáveis & $\begin{array}{c}\text { Razão de } \\
\text { prevalência }\end{array}$ & Erro padrão & $\boldsymbol{p}$-values & IC 95\% \\
\hline Aleitamento materno & 2,5 & 1,02 & 0,030 & $1,08-5,56$ \\
Idade $>$ 48 meses & 1,7 & 0,45 & 0,048 & $1,01-2,85$ \\
\hline
\end{tabular}

\section{Discussão}

O presente estudo identificou a prevalência de excesso de peso em pré-escolares de Teresina, Piauí, Brasil, em 2016, e a sua associação com o aleitamento materno. O resultado encontrado neste estudo foi um pouco superior aos dados registrados no SISVAN para a cidade de Teresina no mesmo período, em que a prevalência de excesso de peso foi de 10,9\% na faixa etária de 2 a 5 anos, segundo o IMC/Idade. ${ }^{(2)}$ Ressalta-se que a cobertura do SISVAN no referido município é em torno de $53 \%$ e que o referido programa é alimentado, principalmente, com os dados dos beneficiários dos programas sociais de transferência de renda.

Pesquisa ${ }^{(21)}$ que avaliou pré-escolares de 2 a 4 anos de idade durante os anos de 2009, 2010 e 2011, em creches do município de Taubaté-SP, encontrou uma prevalência de excesso de peso de $9,3 \%$, valor bem próximo ao encontrado no presente estudo. Por outro lado, outros levantamentos, realizados em diversas cidades do Brasil, apresentam resultados diferentes. Citam-se pesquisas realizadas na Bahia, ${ }^{(22)}$ em Santa Catarina e no Rio Grande do Sul, ${ }^{(23)}$ cujas prevalências de excesso de peso foram de $5,7 \%, 7,5 \%$ e $14,4 \%$, respectivamente. Tal fato mostra diferenças na magnitude do excesso de peso entre os estados brasileiros ${ }^{(21-23)}$ e reforça a necessidade de pesquisas contínuas dessa natureza. Apesar dessas discrepâncias nas prevalências entre as cidades brasileiras, é possível afirmar que houve aumento de crianças com excesso de peso no Brasil, conforme foi 
demonstrado em estudo, ${ }^{(24)}$ a partir da análise de três inquéritos populacionais realizados no País, no período de 1989 a 2006, em que foi evidenciado um aumento de $160 \%$ no excesso de peso nesse período.

No cenário internacional, um estudo censitário e de evolução temporal, que analisou dados de 144 países, estimou haver 43 milhôes de crianças com sobrepeso ou obesidade em 2010, dos quais 35 milhóes estavam nos países em desenvolvimento e ainda demonstrou aumento na prevalência de 4,2\% em 1990 para 6,7\% em 2010. ${ }^{(25)}$ Diante desse contexto, é provável que tais discrepâncias das prevalências do excesso peso nos diferentes cenários seja devido ao processo da transiçáo nutricional em curso no Brasil, sendo determinado, principalmente, pelos fatores socioeconômicos distintos nos estados. ${ }^{(26)}$

No tocante ao aleitamento materno, constatou-se elevada proporção de mães que amamentaram seus filhos, e isso corrobora tanto com o resultado encontrado na II Pesquisa de Prevalência de Aleitamento Materno nas Capitais Brasileiras e Distrito Federal ${ }^{(8)}$ para a cidade de Teresina quanto em relação a pesquisa $^{(15)}$ realizada em Minas Gerais, com proporçóes de $95,2 \%$ e $82,49 \%$ de mães que amamentaram seus filhos por algum período, respectivamente.

No que se refere ao aleitamento materno exclusivo, observou-se baixa prevalência nos menores de 6 meses de idade em relação ao preconizado pela OMS. (7) Os resultados encontrados assemelham-se também com o encontrado na II Pesquisa de Prevalência de Aleitamento Materno nas Capitais Brasileiras e Distrito Federal ${ }^{7}$ para a cidade de Teresina, em que a prevalência do AME foi apenas de 43,7\%. Ademais, outros estudos - como o realizado por Caldeira et al., ${ }^{(15)}$ com pré-escolares em escolas de um município do Estado de Minas Gerais; e o estudo ${ }^{(16)}$ realizado com pré-escolares do município de TaubatéSP - constataram prevalências bem menores que $32,1 \%$ e $25 \%$, respectivamente, em relação ao presente estudo. Dessa forma, percebe-se que os indicadores de aleitamento materno exclusivo ainda permanecem abaixo das recomendaçóes oficiais em diferentes cidades brasileiras, refletindo uma tendência no país.

$\mathrm{Na}$ literatura internacional, pesquisa ${ }^{(27)}$ realizada com escolares em um Centro de Saúde em Lisboa, Portugal, mostrou prevalência de aleitamento materno exclusivo de $28,8 \%$, resultado inferior em relação ao encontrado nesse estudo. Em nível local, estudo ${ }^{(28)}$ que avaliou os fatores associados ao aleitamento materno exclusivo em Hospitais Amigos da Criança (HAC), na cidade de Teresina-PI, encontrou prevalência de 60,49\%. Esse resultado, provavelmente, deve-se ao fato de que as crianças tinham acompanhamento multiprofissional em ambulatório especializado nesses hospitais. Entretanto, vale ressaltar que muitas mães realizaram o desmame precoce de seus filhos antes mesmo do preconizado pela OMS. ${ }^{(7)}$

A regressão de Poisson, mesmo após o controle das variáveis sociodemográficas, demonstrou associação significativa entre aleitamento materno e excesso de peso, mostrando que o ato de não amamentar aumenta a chance desse agravo nas crianças estudadas. Tal achado também foi verificado em outras pesquisas em diferentes contextos e utilizando o mesmo delineamento, como em Minas Gerais ${ }^{(15)}$ e em Lisboa (Portugal). ${ }^{(27)}$ Entretanto, pesquisas realizadas na Região Semi-Árida do Estado de Alagoas ${ }^{(29)}$ e em Pelotas-RS ${ }^{(30)}$ não evidenciaram tal associação; entretanto, em outro estudo realizado nos Estados Unidos, ${ }^{(31)}$ pré-escolares que haviam sido amamentados apresentavam risco reduzido de serem obesos, porém não foi observada essa proteção contra o sobrepeso.

Assim, diante desse cenário, é complexo fazer comparação com esses resultados tão discrepantes. Provavelmente, isso se deve às diferentes metodologias aplicadas nesses estudos, tais como: cálculo e seleçáo da amostra, forma de coleta de dados e instrumentos utilizados, entre outros. Vala a pena ressaltar o viés de memória das mães por se tratarem de dados coletados de forma retrospectiva, como é o caso da prática do aleitamento materno. ${ }^{(18)}$

Contudo, apesar dessas diferenças, em uma revisão sistemática ${ }^{(32)}$ foi demonstrado o papel que a amamentação desempenha na redução da prevalência de obesidade mais tardiamente, os quais incluem mecanismos comportamentais e hormonais e diferenças na ingestão de macronutrientes, dentre os achados vale destacar: uma alta concentração de insulina plasmática em crianças alimentadas com fórmula em comparação as crianças amamentadas levando ao desenvolvimento precoce de adipócitos; a ingestão de proteínas e a quantidade de metabolização energética são meno- 
res em crianças amamentadas do que em crianças alimentadas com fórmula; associação significativa entre a ingestáo precoce de proteínas no início da vida e o aumento risco de obesidade posterior. Ainda foi reportado que a disponibilidade de proteína durante o desenvolvimento fetal, e pós-natal precoce, mostrou ter um efeito a longo prazo na programação metabólica do metabolismo da glicose e na composição corporal futura. Essas vias sozinhas ou combinadas fornecem explicaçôes plausíveis para um efeito protetor do aleitamento materno contra a obesidade. ${ }^{(33)}$

Ainda, nesse estudo, após o controle das variáveis sociodemográficas, demonstrou-se que as crianças maiores de 4 anos apresentaram mais chance de ter excesso de peso em relação aos menores de 4 anos, embora essa questão não tenha sido objeto dessa pesquisa. Tal achado sugere que as crianças com idade inferior a 4 anos são menos vulneráveis ao excesso de peso devido a sua maior dependência para com os pais ou responsáveis, ao maior controle sobre a alimentação servida e também à proteção oferecida pelo aleitamento materno. ${ }^{(3,15,27)}$ Corroborando ainda esse estudo, pesquisa ${ }^{(18)}$ realizada no município de São Paulo com crianças de 2 a 6 anos mostrou que crianças com idade superior a 4 anos teve risco elevado para o excesso de peso. Para esses autores, isso se deve, provavelmente, à maior autonomia na escolha dos alimentos que desejam ingerir, dando preferência aos alimentos com maior valor calórico; além disso, tem-se a facilidade de acesso à mídia e o maior tempo de exposição a televisão e jogos eletrônicos, o que, consequentemente, aumenta o sedentarismo.

Ademais, mostram-se viáveis as intervençôes em centros de educação infantil para abordar a promoção da alimentação saudável e aleitamento materno até os dois primeiros anos de vida. Nesse sentido, cabe mencionar a importância das Escolas Promotoras de Saúde, iniciativa encabeçada pela Organização PanAmericana da Saúde (OPAS) ${ }^{(33)}$ como uma estratégia direcionada, integral e integradora visando o fornecimento de serviços de saúde em ambiente escolar que transcendam a atenção médica tradicional e se fundamentem em açôes de promoção da saúde. Também cabe mencionar a capacidade de integração com estratégias ministeriais, como o Programa Saúde na Escola, para aproximar cada vez mais os pais, os escolares e a escola e assim ter melhores resultados em saúde. ${ }^{(34)}$
O presente estudo trouxe como contribuição a elaboração de um diagnóstico sobre o estado nutricional das crianças na fase pré-escolar e os fatores associados com a ocorrência do excesso de peso. Trata-se do primeiro estudo realizado na cidade de Teresina com essa temática e que poderá contribuir na elaboração de estratégias para a redução desse agravo na infância.

Contudo, apresentou limitações, entre as quais se destaca o fato de a coleta das informaçôes acerca da duração do aleitamento materno ter sido feita retrospectivamente, baseada nas recordaçóes da mãe sobre acontecimentos ocorridos entre três e cinco anos antes, o que pode propiciar um viés de memória. Acresça-se a isso o fato de que o estudo foi realizado apenas em escolas públicas, não permitindo, portanto, maiores generalizaçóes sobre a prevalência do excesso de peso em outras crianças na mesma faixa etária.

\section{Conclusão}

$\mathrm{O}$ aleitamento materno mostrou-se como fator de proteção contra o excesso de peso entre os pré-escolares. Desse modo, é imprescindível adotar medidas de incentivo à promoção do aleitamento materno e à alimentação saudável, além de oferecer atenção integral à criança com excesso de peso, com vistas a reduzir esse agravo e as suas consequências nessa população vulnerável.

\section{Colaborações}

Macêdo RC, Ramos CV, Paiva AA, Martins MCC, Almeida CAPL e Paz SMRS contribuíram com as etapas de concepção do estudo, análise, interpretação dos dados, redação do artigo, revisão crítica relevante do conteúdo intelectual e aprovaçáo final da versão a ser publicada.

\section{Referências}

1. Hart LM, Damiano SR, Cornell C, Paxton SJ. What parents know and want to learn about healthy eating and body image in preschool children: a triangulated qualitative study with parents and Early Childhood Professionals. BMC Public Health. 2015;15:596. 
2. Monasta L, Batty GD, Cattaneo A, Lutje V, Ronfani L, Van Lenthe FJ, Brug J. Early-life determinants of overweight and obesity: a review of systematic reviews. Obes Rev. 2010 0ct;11(10): 695-708.

3. World Health Organization (WHO). Obesity and overweight. Genève: WHO; 2013. Fact Sheet No. 311.

4. Brasil. Ministério da Saúde. Sistema de Vigilância Alimentar e Nutricional (SISVAN). Relatórios públicos do SISVAN [Internet]. Brasília (DF): Ministério da Saúde; 2018. [citado 2018 Ago 9]. Disponível em: http://dabsistemas.saude.gov.br/sistemas/sisvan/login.php?

5. Sociedade Brasileira de Pediatria (SBP). Obesidade na Infância e na Adolescência: manual de orientação para a alimentação do lactente, do pré-escolar, do escolar, do adolescente e na escola. Departamento de Nutrologia, 3a ed. Rio de Janeiro: SBP; 2012. 148 p.

6. Victora CG, Horta BL, Mola CL, Quevedo L, Pinheiro RT, Gigante DP. Association between breastfeeding and intelligence, educational attainment, and income at 30 years of age: a prospective birth cohort study from Brazil. Lancet Global Health. 2015;3(4):e199-e205.

7. World Health Organization (WHO). Report of the expert consultation on the optimal duration of exclusive breastfeeding: conclusions and recommendations. Genève: WHO; 2001.

8. Brasil. Ministério da Saúde. Secretaria de Atenção à Saúde. Departamento de Ações Programáticas e Estratégicas. II Pesquisa de Prevalência de Aleitamento Materno nas Capitais Brasileiras e Distrito Federal. Brasília (DF): Ministério da Saúde; 2009.

9. Assis KF, Oliveira FC, Ribeiro AQ, Araújo RM, Cury AF, Priore SE, et al. Determinantes do abandono do aleitamento materno exclusivo: fatores psicossociais. Rev Saúde Pública. 2014;48(6):985-94

10. Hekmatpou D, Poorgharahkhan A, Sajjadi M, Javaheri A. Effectiveness of the "Create Sensitivity" Caring Model on Blood Glucose/ glycosylated hemoglobin and quality of life in patients with type 2 diabetes. Open Nurs J. 2018;12:195-204.

11. Prado CV, Fabbro MC, Ferreira Gl. Desmame precoce na perspectiva de puérperas: uma abordagem dialógica. Texto Contexto Enferm. 2016; 25(2):e1580015.

12. Yan J, Liu L, Zhu Y, Huang G, Wang PP. The association between breastfeeding and childhood obesity: a meta-analysis. BMC Public Health. 2014;14:1267.

13. Prefeitura Municipal de Teresina. Secretaria Municipal de Educação. Estatísticas [Internet],Teresina: Prefeitura Municipal; 2015. [citado 2019 Out 16]. Disponível em: www.semec.pi.gov.br.

14. Levin J. Estatística aplicada a ciências humanas. 2a ed. São Paulo: Harbra; 1987.

15. Caldeira KM, Souza JM, Sonia B. Excesso de peso e sua relação com a duração do aleitamento materno em pré-escolares. Rev Bras Crescimento Desenvolv Hum. 2015 25(1):89-96.

16. World Health Organization (WHO). Child growth standards: Length/ height- for-age, weight-for-age, weight-for-length, weight-for-height and body mass index-for- age. 1th ed. Genève: WHO; 2006. [cited 2019 Oct 16]. Available from: http://www.who.int/childgrowth/ standards/technical_report/en/

17. Brasil. Ministério da Saúde. Secretaria de Atenção à Saúde. Departamento de Atenção Básica. Orientações para a coleta e análise de dados antropométricos em serviços de saúde: Norma Técnica do Sistema de Vigilância Alimentar e Nutricional - SISVAN. Brasília (DF): Ministério da Saúde, 2011.
18. Simon VG, Souza JM, Souza SB. Aleitamento materno, alimentação complementar, sobrepeso e obesidade em pré-escolares Rev Saúde Pública. 43(1):60-9.

19. World Health Organization (WHO). Indicators for assessing infant and young child feeding practices da WHO [Internet]. 2007. [cited 2019 Oct 16]. Available from: http://apps.who.int/iris/ bitstream/10665/43895/1/9789241596664_eng.pdf.

20. Coutinho LM, Scazufca M, Menezes PR. Methods for estimating prevalence ratios in cross-sectional studies. Rev Saúde Pública. 2008;42(6):992-8.

21. Nascimento VG, da Silva Janaína PC, Ferreira PC, Bertoli CJ, Leone C. Aleitamento materno, introdução precoce de leite não materno e excesso de peso na idade pré-escolar. Rev Paul Pediatr. 2016; 34(4): 454-9.

22. Gomes AT, Novaes TG, Silveira KC, Souza CL, Lamounier JA, Netto MP, Capanema FD, Rocha DS. Excesso de peso e fatores associados em pré-escolares do sudoeste da Bahia. Recife. Rev Bras Saúde Matern. Infant. 2017; 17 (2): 375-83.

23. Schuch I, Castro TG, Vasconcelos FAG Dutra CL, Goldani MZ. Excess weight in preschoolers: prevalence and associated factors. J Pediatr. 2013;89(2):179-88.

24. Silveira JC, Colugnati FA, Cocetti M, Taddei JA. Tendência secular e fatores associados ao excesso de peso entre pré-escolares brasileiros: PNSN1989, PNDS-1996 e 2006/07. J Pediatr (Rio J). 2014; 90( 3 ): 258-66

25. Onis $M$, Blössner $M$, Borghi E. Global prevalence and trends of overweight and obesity among preschool children. Am J Clin Nutr. 2010;92:1257-64.

26. Souza EB. Transição nutricional no Brasil: análise dos principais fatores. Cadernos UNIFOA. Edição n. 13, Agosto; 2010.

27. Ferraria N, Rodrigues V, Macedo L. Aleitamento materno e excesso de peso em crianças na idade escolar. Scient Méd. 2013; 23(2): 75-81.

28. Ramos CV, Almeida JA, Saldiva SR, Pereira LM, Alberto NS, Teles JB. Prevalência do aleitamento materno exclusivo e os fatores a ele associados em crianças nascidas nos Hospitais Amigos da Criança de Teresina - PI. Epidemiol Serv Saúde. 2010;19(2):115-24.

29. Moreira MA, Cabral PC, Ferreira HS, Lira PIC. Excesso de peso e fatores associados em crianças da região nordeste do Brasil. J Pediatr. 2012; 88(4):347-52.

30. Araúijo CL, Victora CG, Hallal PC, Gigante DP. Breastfeeding and overweight in childhood: evidence from the Pelotas 1993 birth cohort study. Int J Obes (Lond). 2006;30:500-6.

31. Hediger ML, Overpeck MD, Kuczmarski RJ, Ruan J. Association between infant breastfeeding and overweight in young children. JAMA. 2001;285:2453-60.

32. Arenz S, Rückerl R, Koletzko B, Von Kries R. Breast-feeding and childhood obesity--a systematic review. Int J Obes Relat Metab Disord. 2004;28(10):1247-56

33. Organização Panamericana de Saúde (OPAS) - Brasil. Escolas Promotoras de Saúde: relatório técnico. OPAS; 2018. Available from: https://www.paho.org/bra/index.php?option=com_content\&view=arti cle\&id=588:escolas-promotoras-de-saude\&ltemid=685

34. Brasil. Ministério da Saúde. Programa Saúde na Escola [Internet]. [citado 2019 Out 16]. Disponível em: http://www.saude.gov.br/acoese-programas/programa-saude-na-escola 